\title{
Importance of a Psychological Autopsy to Identify the Manner of Death; a Case of fall from Height
}

\author{
Sathasivam Raveendran*, Muditha Vidanapathirana \\ Department of Forensic Medicine, Faculty of Medical Sciences, University of Sri Jayewardenepura, Sri Lanka.
}

\begin{abstract}
It may not be possible to determine the manner of death, in circumstances such as death following fall from height. When a dead body is found in front of a tall building, the ascertainment of the manner of death is difficult and in this case, the importance of a psychological autopsy in such circumstance is discussed. Middle-aged female with bronchial asthma, arterial hypertension, chronic venous insufficiency, Forestier disease, hyperlordosis of lumbar spine and vitamin D deficiency developed bilateral leg swelling and rash following 14 hours of air travel. Duplex scan excluded lower limb venous thrombosis. She did not have past history of psychiatric disorder but suddenly developed aggressive abnormal behaviour, confusion and bizarre activities. She removed her clothes and finally jumped off from 6th floor of her residency. She was succumbed to death due to multiple injuries following fall from height. No suicidal letter was recovered from the scene. The manner of death was ascertained as suicide based on eyewitness evidence. Her sudden onset of abnormal and violent behaviour may be an act of automatism without volitional control due to undiagnosed inorganic or organic disease of the brain. The witnessed suicidal behaviour was reverted to accident as a manner of death. The importance of psychological autopsy and teamwork between Forensic Pathologist and Forensic Psychiatrist in suspicious circumstances to ascertain the manner of death is highlighted. Labelling as suicide will end up in negative social stigma and loss of insurance claims.
\end{abstract}

Keywords: Automatism, brain, fall from height, manner of death, psychological autopsy

Received: 02 April 2019, Revised version accepted: 20 June 2019, Published: 30 June 2018. *Corresponding author: Raveendran S, \Email: sathasivamraveendran10@gmail.com, (D) https://orcid.org/0000-0002-2313-6491

Cite this article as: Raveendran S, Vidanapathirana M. Importance of a Psychological Autopsy to Identify the Manner of Death; a Case of fall from Height. Medico-Legal Journal of Sri Lanka, 2019;7(1): 32-6. DOI: http://doi.org/10.4038/mljsl.v7i1.7392

Copyright: @ 2019 with the Medico-legal Journal of Sri Lanka.

This is an open-access article distributed under the terms of the Creative Commons Attribution 4.0 International License, which permits unrestricted use, distribution and reproduction in any medium provided the original author and source are credited.

\section{Introduction}

It may not be possible for the inquirer into sudden deaths/magistrate, in a best good-faith judgment, to determine the exact manner of death, in circumstances such as death following fall from height. In such instances, the manner of death may be ascertained as "undetermined". ${ }^{[1]}$ Fall from height is defined as an injury to a person that occurs after landing on the ground after falling from a higher place. ${ }^{[2]}$ For example, person may fall from a ladder, scaffold, building, roof or other elevated place or work area. In such circumstances, the ascertainment of the manner of death may be difficult without performing a psychological autopsy, preferably by a forensic psychiatrist. Psychological autopsy is one of the most valuable tools of research on completed suicide. The method involves collecting all available information on the deceased via structured interviews of family members, relatives or friends as well as attending health care personnel. In addition, information is collected from available health care and psychiatric records, other documents, and forensic examination. ${ }^{[3]}$ The importance of a psychological autopsy to ascertain the manner of death in such circumstance is discussed in this case.

\section{Case report}

A body of a 49-year-old women was found on the ground floor of a sixth storied apartment in Colombo. She was a citizen of a foreign country and came to Sri Lanka as a tourist after travelling 14 hours in the flight. Red coloured rash and swelling were developed in her both legs following the arrival to Sri Lanka. Four days later, she consulted a vascular surgeon and a duplex scan revealed that venous system of both lower limbs were normal. She was confused and severely disturbed due to her illness and she had not slept adequately due to frequent urination. She had cried frequently in the previous day of the death. On the day of incident, she suddenly developed aggressive behaviour and became 
confused. A domestic servant said that the deceased was talking herself about her body parts and acting like a film star in front of a mirror. Thereafter, she had started removing her clothes one by one and had thrown the clothes outside the balcony. When the domestic servant tried to prevent her behaviour, deceased behaved aggressively and jumped off from the balcony of the sixth floor. Her past medical records revealed that she had bronchial asthma, arterial hypertension, chronic venous insufficiency, Forestier disease, hyperlordosis of lumbar spine and lack of vitamin $\mathrm{D}$. The deceased has had arthritis and chronic venous insufficiency.

The dead body was found naked in prone position on the cemented floor in front of the 6 storied building (Fig. 01).

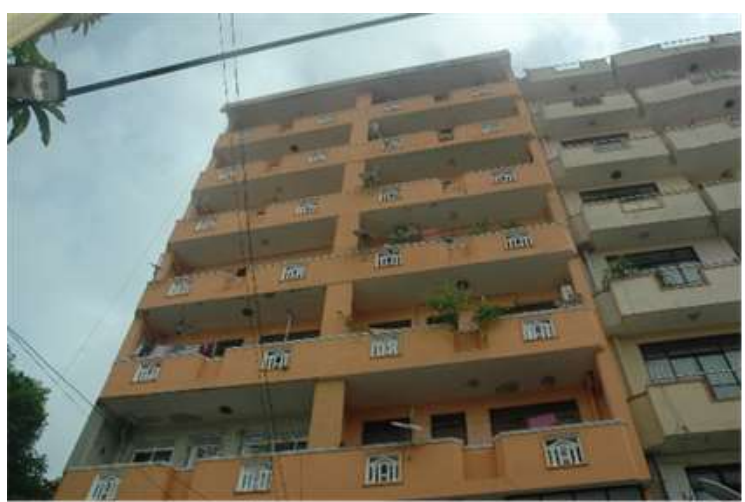

Figure 01: The six storied building

A brassiere, blouse, panty, underskirt, frock and some other clothes were also found on the ground floor in front of the building. A blue coloured intervening metal ceiling sheet showed few indentations. (Fig. 02).

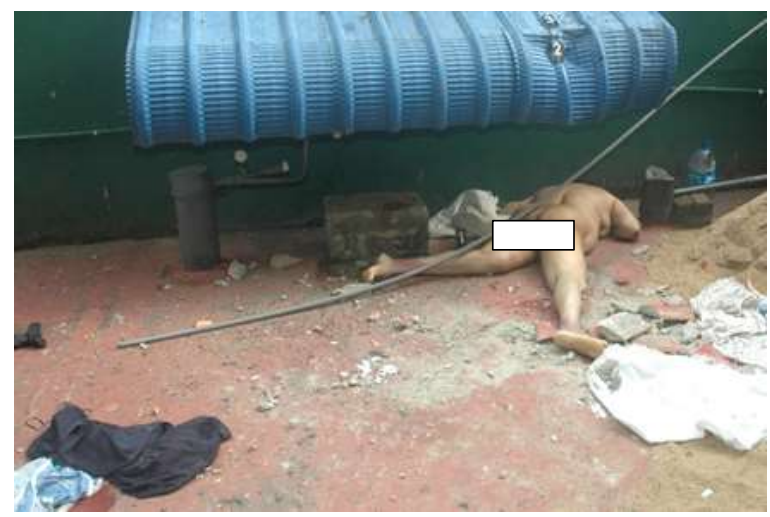

Figure 02: Blue coloured intervening metal object had indentations.

She did not have family history of suicides or psychiatric illness and had not have suicidal thoughts or previous suicidal attempts. No suicidal letter was recovered from the scene. The clothes were not torn.

The autopsy revealed a well-preserved body and she was average built and nourished. The deceased had a rash and ankle swelling in both legs (Fig. 08). There were no red or pale patches or frostbites.

There were no healed scars or deformities. There were abrasions and minor contusions on the scalp, extensive brush burns and contusions on the front aspect of the body, stretch lacerations of left inguinal region, multiple extensive bilateral rib fractures at several places and transection of the thoracic vertebral column at T-12 level. No skull fractures (Fig. 03, 04 and 05), extradural, subdural and subarachnoid haemorrhages were detected at the autopsy.

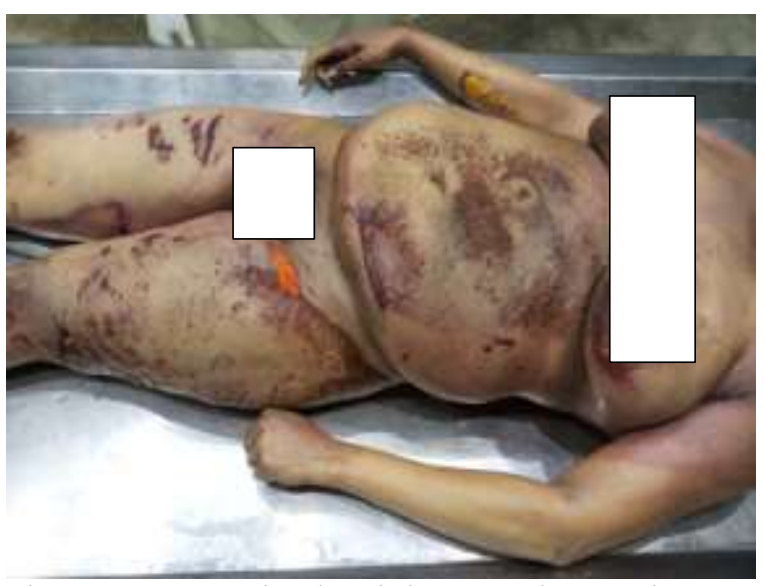

Figure 03: Extensive brush burns and contusions on the front aspect of the body

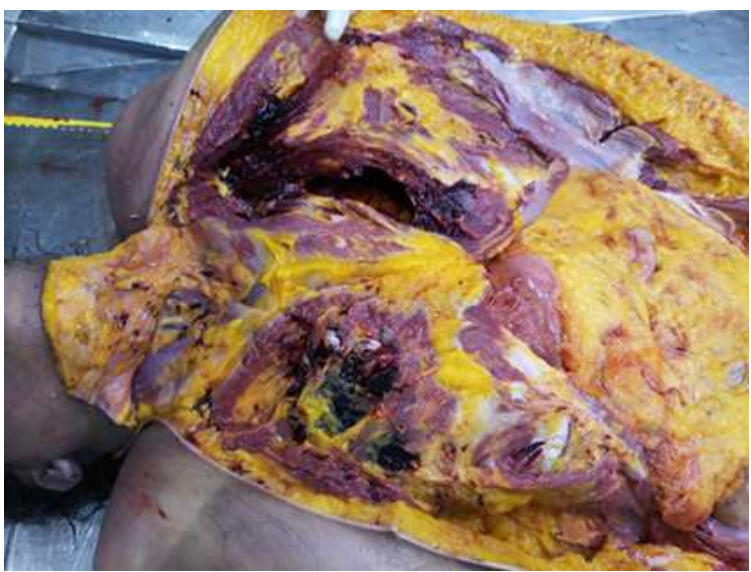

Figure 04: Multiple extensive bilateral rib fractures 


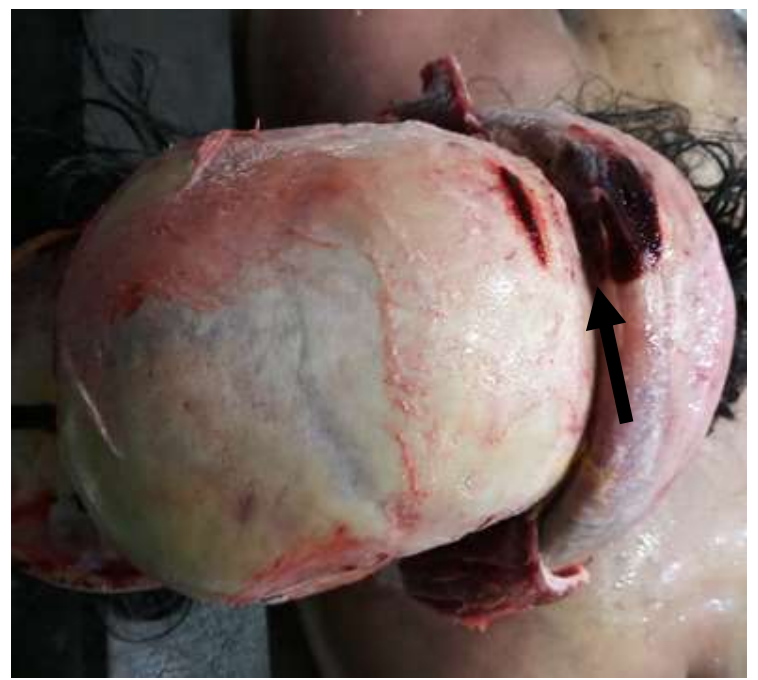

Figure 05: Contusions on the scalp. There were no skull fractures.

Brain was macroscopically and microscopically unremarkable. The pericardium was ruptured completely and clotted blood was found around it. The right atrium and left ventricle of the heart were lacerated. Coronary arteries were patent. Aorta was transected and separated just below the arch (Fig. 06). Both lungs were lacerated at the hilum and collapsed.

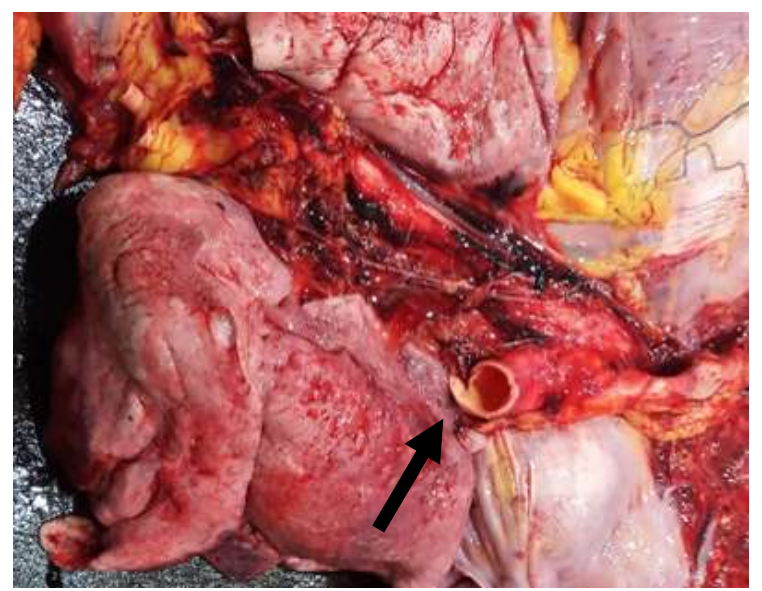

Figure 06: Transection of the aorta

Peritoneal bleeding was not significant. No injuries were detected at diaphragm. The liver was pulped with multiple lacerations. (Fig. 07). Right kidney was lacerated with surrounding haemorrhagic contusion.

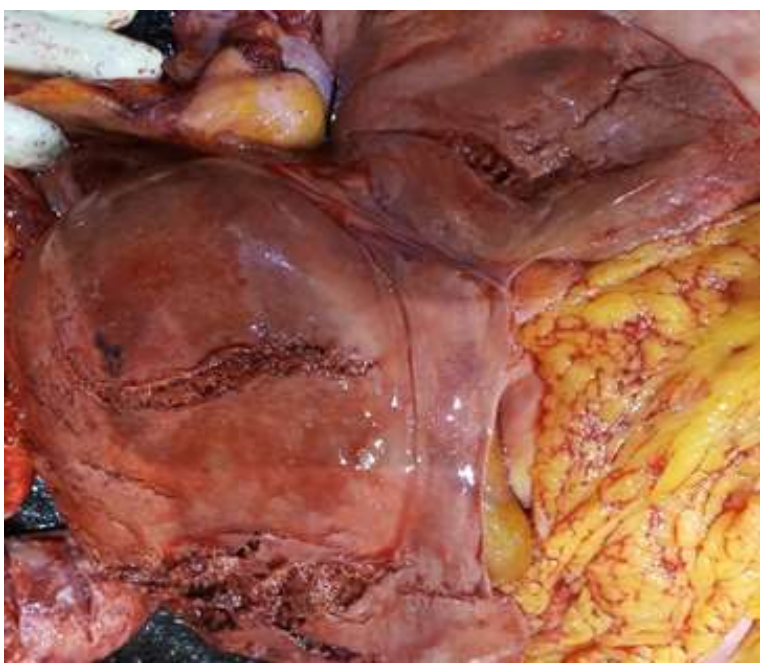

Figure 07: Pulped liver with multiple lacerations

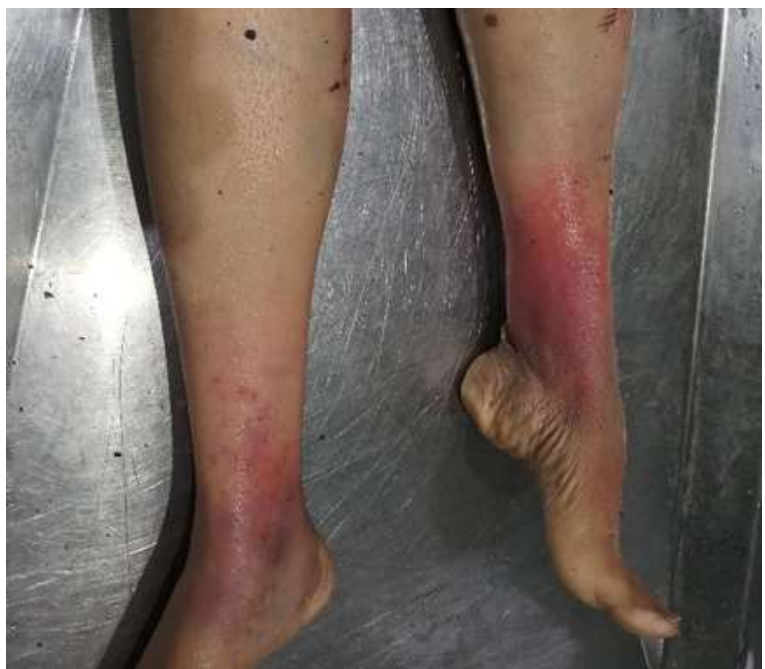

Figure 08: Arthritis, rash and ankle swelling in both legs

\section{Discussion}

Without doing a psychological autopsy, a case should not have been concluded as suicide, because it can create a social stigma to the deceased and the family. Further, the next of kin will not be able to get full insurance claims. ${ }^{[1]}$ Moreover, reliable clinicopathological analysis on the basis of pathology and crime scene alone, not always possible in predicting the kind and degree of psychic symptoms observed. This issue is occasionally raised in the forensic arena, where a violent or habitual criminal is suspected of having or discovered to have some form of focal brain pathology. ${ }^{[4]}$

In this case, the presence of extensive brush burns and contusions on the front aspect of the body, stretch lacerations of left inguinal region, multiple extensive bilateral rib fractures at different places and 
transection on thoracic vertebral column at T-12 level suggested that the deceased had suffered blunt force injuries. The presence of the transection of the aorta just after the arch, bilateral collapsed and lacerated lungs, kidney lacerations, multiple liver lacerations with minimal peritoneal bleeding suggested that these were due to sudden deceleration following a fall from height. ${ }^{[5]}$ It was further confirmed by the presence of indentations in the metal intermediate object and the independent eyewitness evidence.

The deceased had abrasions and contusions of the soft tissues of the head, however, there were no skull fractures or meningeal/cerebral haemorrhages. Her initial fall stopped as she hit the blue shed. In that probably she did not hit her head. Then from there she had fallen to the ground, which is a small fall. Lack of skull fractures, meningeal/cerebral haemorrhages and brain injuries were unusual when compared with earlier reported cases of fall from height. ${ }^{[6,7]}$ The brain was congested and had no macroscopic injuries. But the injuries of the brain cannot be excluded completely because of the nature of quick death and there was no time for appearance of ante-mortem evidence of diffuse brain injuries/severe diffuse axonal injuries. ${ }^{[6]}$ Further, the deceased had chest injuries, abdominal injuries, lower limb and upper limb injuries. Therefore, the cause of death was given as multiple injuries to the body due to blunt force injuries and the injury pattern was consistent with a fall from height.

Then the important medico-legal issue in this case was the manner of death; whether it was a homicide, suicide or accident? The deceased was living happily and returned homeland to see her relatives and the native places. According to the history from relatives and witness, there had been no reasons to commit suicide. The deceased had jumped out through the balcony of the sixth floor and it had been witnessed by the domestic servant. It was compatible with an act of suicide when it is analysed superficially. It may be further supported by her illness and depressive symptoms such as loss of sleep and crying before the incident.

Her body was found naked and she had removed her clothes before jumping off from her $6^{\text {th }}$-floor residence. Paradoxical undressing with hide and die syndrome in hypothermia was excluded due to the absence of associated other features such as red or pale patches or frostbites. The victims, despite low environmental temperatures, paradoxically remove their clothes due to a sudden feeling of warmth. The temperature of the environment was warm. ${ }^{\left[{ }^{8]}\right.}$ Further, she had behaved in a confused manner before the act of jump. These are compatible with an acute psychotic behaviour. Therefore, her sudden onset of abnormal and violent behaviour may be an act of automatism without volitional control due to undiagnosed inorganic or organic disease of the brain. ${ }^{[9]}$

The deceased had arthritis and rash on both legs and chronic venous insufficiency (Fig. 08). It may be associated with various types of vasculitis and autoimmune disorders such as Systemic lupus erythematosus (SLE) and multiple sclerosis. It may be the other cause for transient hypoxia to the brain or autoimmune inflammatory encephalopathy. These are called autoimmune brain diseases. ${ }^{[10]}$ It might not have been diagnosed earlier. Such illness may also be a reason for her sudden provocation with psychotic symptoms and self-motivated act of jump off from height.

According to the forensic psychiatrist's opinion, the different types of psychiatric disorders can cause depression, aggressive behaviour, bizarre type of activities and suicidal tendencies. If above disorders are excluded safely, then the psychogenic, organic and non-organic automatism induced behaviour can be considered as the reason for her jump. These are beyond the scope of the forensic pathologist, however, it can be diagnosed by performing a retrospective psychological autopsy by a forensic psychiatrist, though it is not a routine practice in Sri Lanka. If the act was due to an automatism caused by an organic disease, it should be detected by the Forensic Pathologist by a thorough autopsy investigation including multiple sections of the brain with special stains and immune histochemistry. ${ }^{[1]}$ However, except $\mathrm{H}$ and $\mathrm{E}$, these tests were not done in this case due to lack of facilities. Therefore, such meticulous autopsy investigation is important for the Forensic psychiatrist to interpret automatism.

Kolle SR et al reported a case of 21 years old man succumbed to the injuries following fall from threestory building with homicidal or suicidal act suspected by relatives and police, but later it was concluded as an accident due to somnambulistic automatism. ${ }^{[11]}$ Therefore, the importance of a retrospective psychological autopsy in such circumstances is reiterated for the Forensic pathologist to reach the final conclusion regarding the manner of death. Finally, this case was reverted to accident as the manner of death by the magistrate.

\section{Conclusions}

The witnessed suicidal behaviour was reverted to accident as a manner of death. Further, the importance of Psychological Autopsy and teamwork between Forensic Pathologist and Forensic Psychiatrist 
especially in suspicious circumstances to ascertain the manner of death is highlighted. Labelling as suicide will end up in negative social stigma and loss of insurance claims.

\section{Disclosure statement}

Conflicts of interests: The authors declare that they have no conflicts of interests.

Funding: None

\section{References}

1. Leestma JE, Sharp EW. Pathology and Neuropathology in the Forensic Setting. In; Leestma J, editor. Forensic Neuropathology. 2nd ed. U.S.A: CRC Press; 2009. 1-25

2. Turgut K, Sarihan ME, Colak C, Guven T, Gur A, Gurbuz S. Falls from height: A retrospective analysis. World J Emerg Med. 2018;9(1):46-50. PMC 5717375

3. Isometsa ET. Psychological autopsy studies--a review. Eur Psychiatry. 2001 Nov;16 (7):379-85. PMID: 11728849

4. Leestma JE. Forensic Aspects of Complex Neural Functions. In; Leestma J, editor. Forensic Neuropathology. 2nd ed. USA: CRC Press; 2009. 659-708

5. Buckman RF, Buckman PD. Vertical deceleration trauma: principles of management. Surg. Clin. North. Am. 1991; 71(2):331-44. PMID:2003254
6. Armbrustmacher V, Hirsch CS. Trauma of the Nervous System. In: Spitz WU, Fisher DJ, editors. Medico legal investigation of death. 4th ed. USA: Charles C Thomas Publishers Ltd; 2005. 994-1077

7. Mckee AC, Daneshvar DH. The neuropathology of traumatic brain injury. Handb Clin Neurol. 2015; 127: 45-66. Doi:10.1016/B978-0-44452892-6.00004-0

8. Descloux E, Ducrot K, Scarpelli MP, Lobrinus A, Palmiere C. Paradoxical undressing associated with subarachnoid hemorrhage in a nonhypothermia case? Int J Legal Med. 2017 Sep;131(5):1341-5. Doi: 10.1007/s00414-0171597-3.

9. McCaldon RJ. Automatism. Can Med Assoc J. 1964 Oct 24; 91(17): 914-20. PMID:14199824

10. Matthew SK, Dalmau J. The Emerging Link Between Autoimmune Disorders and Neuropsychiatric Disease. J Neuropsychiatry Clin Neurosci. 2011; 23(1): 90-97. Doi: 10.1176/appi. neuropsych.23.1.90

11. Kolle SR, Chaudhari VA. Extreme complication of somnambulism: death due to accidental fall from height. Int J Res Med Sci 2016; 4(12):5491 3. DOI: http://dx.doi.org/10.18203/2320-6012. ijrms20164237 